\title{
Neurophotonics
}

\section{Voltage-sensitive dye imaging of mouse neocortex during a whisker detection task}

\author{
Alexandros Kyriakatos \\ Vijay Sadashivaiah \\ Yifei Zhang \\ Alessandro Motta \\ Matthieu Auffret \\ Carl C. H. Petersen
}




\title{
Voltage-sensitive dye imaging of mouse neocortex during a whisker detection task
}

\author{
Alexandros Kyriakatos, Vijay Sadashivaiah, Yifei Zhang, Alessandro Motta, Matthieu Auffret, and \\ Carl C. H. Petersen* \\ Brain Mind Institute, Laboratory of Sensory Processing, Faculty of Life Sciences, École Polytechnique Fédérale de Lausanne (EPFL), \\ Lausanne $\mathrm{CH}-1015$, Switzerland
}

\begin{abstract}
Sensorimotor processing occurs in a highly distributed manner in the mammalian neocortex. The spatiotemporal dynamics of electrical activity in the dorsal mouse neocortex can be imaged using voltagesensitive dyes (VSDs) with near-millisecond temporal resolution and $\sim 100-\mu \mathrm{m}$ spatial resolution. Here, we trained mice to lick a water reward spout after a 1-ms deflection of the $\mathrm{C} 2$ whisker, and we imaged cortical dynamics during task execution with VSD RH1691. Responses to whisker deflection were highly dynamic and spatially highly distributed, exhibiting high variability from trial to trial in amplitude and spatiotemporal dynamics. We differentiated trials based on licking and whisking behavior. Hit trials, in which the mouse licked after the whisker stimulus, were accompanied by overall greater depolarization compared to miss trials, with the strongest hit versus miss differences being found in frontal cortex. Prestimulus whisking decreased behavioral performance by increasing the fraction of miss trials, and these miss trials had attenuated cortical sensorimotor responses. Our data suggest that the spatiotemporal dynamics of depolarization in mouse sensorimotor cortex evoked by a single brief whisker deflection are subject to important behavioral modulation during the execution of a simple, learned, goal-directed sensorimotor transformation. ๑ The Authors. Published by SPIE under a Creative Commons Attribution 3.0 Unported License. Distribution or reproduction of this work in whole or in part requires full attribution of the original publication, including its DOI. [DOI: 10.1117/1.NPh.4.3.031204]
\end{abstract}

Keywords: sensory perception; sensorimotor processing; goal-directed sensorimotor transformation; whisker sensation; licking; reward; mouse; neocortex; sensorimotor cortex; voltage-sensitive dye imaging.

Paper 16054SSR received Jul. 31, 2016; accepted for publication Oct. 19, 2016; published online Nov. 23, 2016.

\section{Introduction}

Learned behaviors typically involve context-dependent processing of sensory information based on current motivation and previous experience to convert the sensory information into a useful motor output. Such learned, goal-directed sensorimotor transformations are likely to involve multiple brain areas, including the participation of distinct regions of the neocortex. It is therefore important to investigate the spatiotemporal dynamics of cortical activity during execution of a learned, goal-directed sensorimotor transformation.

Voltage-sensitive dye (VSD) imaging offers the opportunity to measure cortical spatiotemporal dynamics in vivo with approximately millisecond temporal resolution and $\sim 100-\mu \mathrm{m}$ spatial resolution, revealing highly dynamic responses to sensory stimulation with complex spatiotemporal spread. ${ }^{1,2}$ Both spontaneous and evoked VSD signals have been found to correlate well with subthreshold membrane potential fluctuations of simultaneously recorded L2/3 pyramidal neurons in vivo under anesthesia. ${ }^{3-6}$ VSD imaging therefore appears to be useful for imaging the spatiotemporal dynamics of ensemble electrical activity in the mammalian neocortex.

Whisker sensation is important for rodents, ${ }^{7,8}$ and the spatiotemporal dynamics of cortical processing of whisker-related information have previously been studied extensively using VSD imaging in anesthetized animals and in awake animals not trained to perform any task. ${ }^{3-6,9-20}$ The deflection of a single

*Address all correspondence to: Carl C. H. Petersen, E-mail: carl.petersen@ epfl.ch whisker initially evokes a highly localized sensory response, mapping onto the appropriate barrel column in the primary somatosensory cortex (S1). ${ }^{3,5,11}$ However, the sensory response typically spreads over the next milliseconds, invading neighboring cortical barrel columns, depending upon stimulus strength, ${ }^{3,6}$ developmental age, ${ }^{21,22}$ ongoing spontaneous cortical activity, ${ }^{4}$ and ongoing whisker-related behavior. ${ }^{5,13}$ In addition, separate secondary hotspots of activity can be observed within tens of milliseconds of whisker deflection, including prominent depolarization of a frontal cortical region, in mouse located $\sim 1 \mathrm{~mm}$ anterior and $\sim 1 \mathrm{~mm}$ lateral to Bregma, the whisker motor cortex (M1). ${ }^{13,15,16}$ The sensory response in whisker M1 depends upon activity in S1 because it is almost entirely blocked by inactivating $\mathrm{S} 1{ }^{13}$ Whisker M1 contains a functional whisker sensory map, which is compressed and mirror-reflected relative to the $\mathrm{S} 1$ map. $^{13,15}$ Anatomically, S1 axons directly innervate M1 in a columnar somatotopic manner. ${ }^{13,23-27}$ Stimulation of mouse whisker M1 evokes rhythmic whisking. ${ }^{15,28,29}$ Interestingly, the apparent same brain region has been reported to relate to several other aspects of behavior, including orienting, ${ }^{30}$ licking, ${ }^{31}$ and rotor-rod performance. $^{32}$

The dynamic pattern of cortical activity evoked by a sensory stimulus depends on ongoing behavior and current task requirements, and it is therefore of interest to examine the spatiotemporal dynamics of the sensorimotor cortex during task performance. Here, we imaged neocortical function using VSD during a simple sensorimotor task in which a thirsty mouse converts a single brief whisker-deflection into licking for water reward. Previous investigations of closely related 
whisker-dependent detection tasks have found task-dependent correlations in action potential firing and membrane potential dynamics of individual neocortical neurons recorded in $\mathrm{S} 1^{33-36}$ and $\mathrm{M} 1,{ }^{37}$ as well as in striatal projection neurons. ${ }^{38}$ Inactivation of S1 disrupts behavioral performance in whisker-detection tasks, reducing hit rates. ${ }^{33,34,39}$ Stimulation of S1 readily substitutes for whisker stimulation in learning and execution of this task. ${ }^{33} \mathrm{~S} 1$ therefore appears to play a critical role in the sensorimotor transformation of whisker stimulus into licking motor output. It is unlikely that whisker $\mathrm{S} 1$ has a direct motor function with respect to licking, and presumably $\mathrm{S} 1$ functions by sending signals to other parts of the brain. Indeed, S1 projects directly to many other brain regions. ${ }^{25}$ In terms of ipsilateral corticocortical connectivity, S1 prominently, directly, and reciprocally innervates S2 and whisker M1. Because of the lateral aspect of $\mathrm{S} 2$, in this study we were unable to image this cortical region, but all experiments included both S1 and whisker M1. Our results suggest highly distributed sensorimotor processing that depended strongly upon whisking and licking during task performance.

\section{Materials and Methods}

All experimental procedures were approved by the Swiss Federal Veterinary Office.

\subsection{Animals, Surgery, and Behavioral Training}

Mice were implanted with a metal head-restraint post at five to nine weeks after birth under isoflurane anesthesia. All whiskers were trimmed except for the $\mathrm{C} 2$ whiskers on either side. The behavioral training was carried out more or less as previously described, ${ }^{33,35,36,38}$ except that (i) in this study, we had an explicit cue from shutter and imaging light indicating trial onset and (ii) unlike in the previous studies, here we did not abort trials with prestimulus licking. Briefly, water-restricted mice were taught to associate a 1-ms magnetic pulse applied to iron particles attached to the right $\mathrm{C} 2$ whisker delivered $2.5 \mathrm{~s}$ after the trial onset cue with water availability, delivered via a reward spout. A drop of water was delivered if the mouse licked within the reward time window ( 0.1 to $1 \mathrm{~s}$ postwhisker stimulus). Catch trials (no whisker stimulus) were randomly interleaved with whisker stimulus trials to obtain the false-alarm rates. Behavioral control and behavioral data collection were carried out with custom written computer routines using an ITC18 (Instrutech) interfaced through IgorPro (Wavemetrics). After training, mice were subsequently used for a single session of VSD imaging.

\subsection{Voltage-Sensitive Dye Imaging}

A craniotomy over the dorsal cortex was carried out under isoflurane $(1.5 \%)$ anesthesia. Extreme care was taken at all times not to damage the cortex, especially during removal of the dura. The VSD RH1691 was dissolved at $1 \mathrm{mg} / \mathrm{ml}$ in Ringer's solution containing (in $\mathrm{mM}$ ) $135 \mathrm{NaCl}, 5 \mathrm{KCl}$, $5 \mathrm{HEPES}, 1.8 \mathrm{CaCl}_{2}$, and $1 \mathrm{MgCl}_{2}$. This dye solution was topically applied to the exposed cortex and allowed to diffuse into the cortex for 1 to $2 \mathrm{~h}$. The cortex was subsequently washed to remove unbound dye and covered with $1 \%$ agarose before gluing a cover slip on top. The mouse was subsequently returned to its home cage for at least $1 \mathrm{~h}$ to recover. The VSD was excited with $630-n m$ light from a $100-\mathrm{W}$ halogen lamp gated by a Uniblitz shutter (Vincent Associates) under computer control via an ITC18 (Instrutech) communicating with custom software running within IgorPro (Wavemetrics). The excitation light was reflected using a 650-nm dichroic and focused onto the cortical surface with a 50-mm camera lens (Nikon). Fluorescence was collected via the same optical pathway, but without reflection of the dichroic, long pass filtered $665 \mathrm{~nm}$, and focused onto the sensor of a high-speed MiCam Ultima (Scimedia) camera via a 50-mm video lens (Navitar). This high-speed CMOS camera has a detector with $100 \times 100$ pixels. The field of view was $10 \times 10 \mathrm{~mm}$, and therefore every pixel collected light from a cortical region of $100 \times 100 \mu \mathrm{m}$. Images were collected with 2-ms temporal resolution and analyzed offline using custom written routines in IgorPro, Python, and MATLAB ${ }^{\circledR}$. Bleaching of fluorescence was corrected by subtraction of correct rejection trials, i.e., trials without whisker stimulation and without licking. Time courses of fluorescence changes were quantified as $\Delta F / F_{0}$ from regions of interest covering $5 \times 5$ pixels (corresponding to $500 \times 500 \mu \mathrm{m}$ of cortex). To compare VSD signals from different animals, regions of interest were centered on the locations of the earliest responses in S1 and M1. Responses from these functionally identified regions were then compared or averaged across different experiments. The amplitudes of the early sensory-evoked responses were calculated as the change in the VSD signal $\left(\Delta F / F_{0}\right)$ from baseline (an average of the five frames immediately before the stimulus) to the peak of the response (an average of three frames centered upon the maximum of the trial-averaged response). The amplitudes of the late response components were quantified as the change in fluorescence $\left(\Delta F / F_{0}\right)$ comparing the average over 100 to $300 \mathrm{~ms}$ with the prestimulus baseline averaged across trials.

In some experiments, we used high-speed video filming to quantify whisker movements. The mouse was illuminated with infrared light and filmed through a 50-mm video lens (Navitar) with a high-speed camera (Optronis). The behavioral images were obtained at 2-ms intervals between frames synchronized to the VSD imaging through Transistor-transistor logic pulses. Custom-written routines in IgorPro were used to semiautomatically track whisker position.

\subsection{Statistical Analysis}

All values are presented as the median together with the 25th and 75th percentiles of the distribution, except where otherwise stated. Statistical testing was carried out in IgorPro. We used the Wilcoxon signed-rank test for paired data, and the WilcoxonMann-Whitney test for unpaired data. All tests were twosided. The sensitivity index d prime $\left(d^{\prime}\right)$ from signal detection theory was computed as $d^{\prime}=z$ (hit rate) $-z$ (false alarm rate), with the $z$ scores computed in Excel using the function NORMSINV. We applied Bonferoni's correction for multiple comparisons, with differences being considered statistically significant for $P$ values lower than $0.05 / n$, where $n$ is the number of statistical comparisons being carried out.

\section{Results}

\subsection{Voltage-Sensitive Dye Imaging During Task Performance}

Water-scheduled mice were trained to lick a water-reward spout in response to a $\mathrm{C} 2$ whisker deflection evoked by a 1-ms magnetic pulse acting on iron particles attached to the $\mathrm{C} 2$ whisker 
(a)

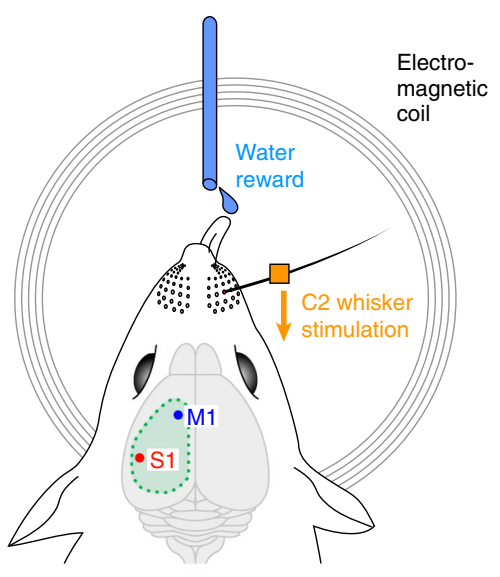

(b)

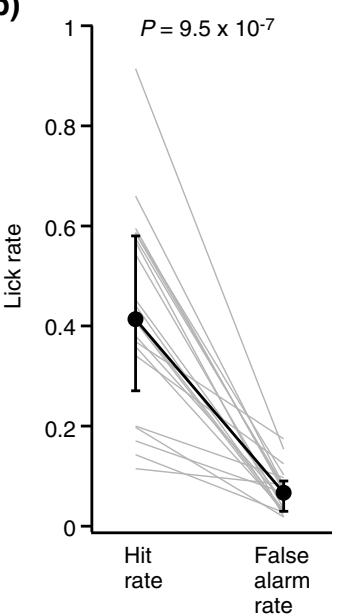

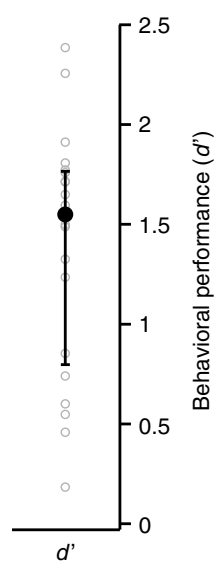

(c) Experiment 121109
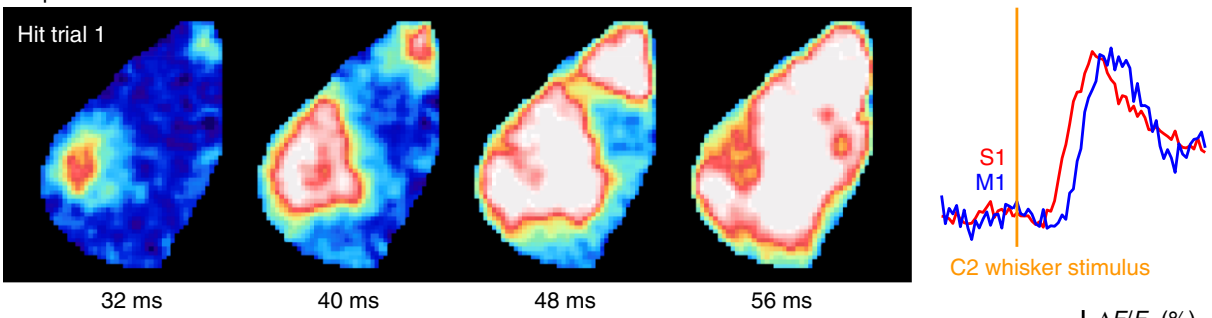

C2 whisker stimulus
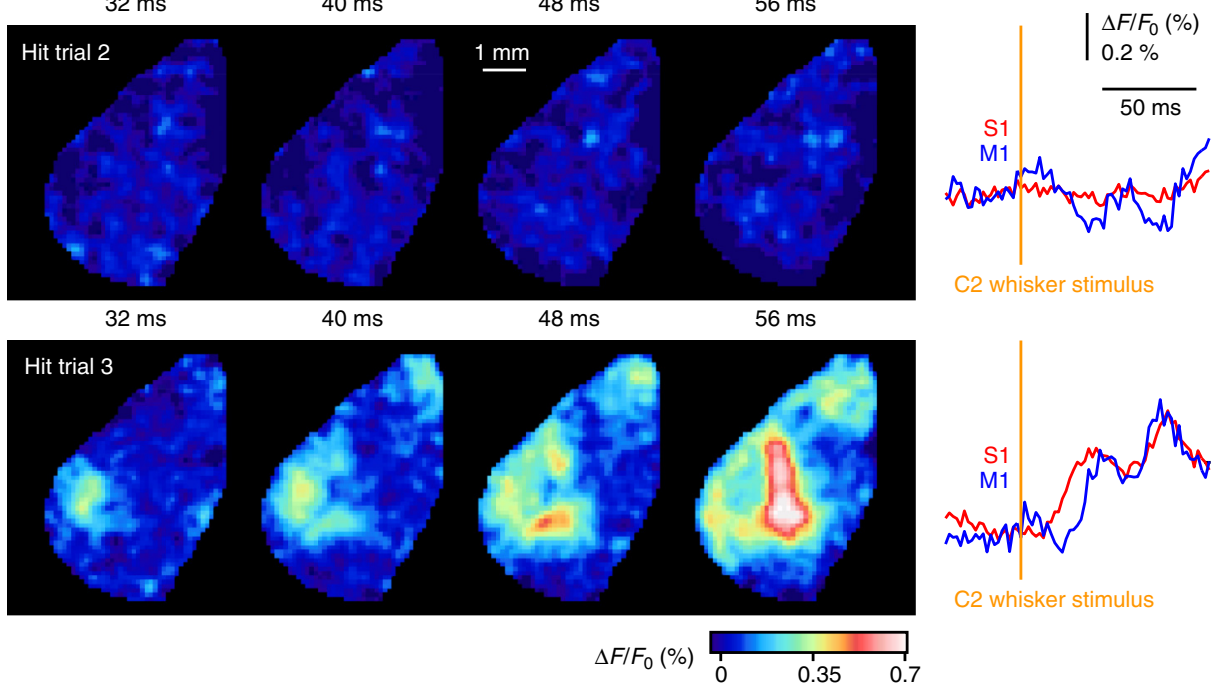

Fig. 1 VSD imaging during a detection task in which a trained thirsty mouse licks a water spout after whisker stimulation to obtain liquid reward. (a) A brief single whisker deflection was evoked by a 1-ms current pulse flowing through an electromagnetic coil located below the head of the mouse, which generated a brief magnetic field acting on iron particles attached to the $\mathrm{C} 2$ whisker. If the thirsty mouse licked a spout in the 1-s period after whisker stimulation, it was rewarded by a small drop of water. (b) In trained mice during VSD imaging, the hit rate (probability of licking in the 1-s reward window) was significantly larger than the false alarm rate (probability of licking in the 1-s catch window) according to the Wilcoxon signed-rank test. (c) Example hit trial images (left) from an individual experiment at early times after whisker stimulation at $0 \mathrm{~ms}$. Time course of fluorescence changes (right) in regions of interest $(500 \times 500 \mu \mathrm{m})$ centered on the C2 whisker responsive regions in S1 (red) and M1 (blue) for the individual trials shown on the left. Responses showed obvious trial-to-trial variability.

[Fig. 1(a)]..$^{33}$ If the mouse licked the spout within $1 \mathrm{~s}$ of the whisker stimulus, a water reward was delivered. The beginning of each trial was indicated by the noise of the shutter opening to illuminate the dorsal neocortex with red light $2.5 \mathrm{~s}$ before the whisker stimulus. Whisker stimulus and no-stimulus catch trials were randomly interleaved. After $\sim 1$ week of $\sim 1$ h daily training sessions, the mice learned to lick in response to the whisker stimulus and to withhold licking on catch trials. In the last training day (the day before VSD imaging), the mice $(n=21)$ performed with a median hit rate of 0.81 (interquartile range 0.65 to 0.85 ), a median false alarm rate of 0.30 (interquartile range 0.18 to 0.36 ), and a median $d^{\prime}$ of 
1.38 (interquartile range 0.89 to 1.98 ). The cortex was then stained with VSD RH1691 and imaged in a single final behavioral session. During the imaging session, the mice $(n=21)$ had a median hit rate of 0.41 (interquartile range 0.27 to 0.58 ), a median false alarm rate of 0.07 (interquartile range 0.03 to 0.09 ), and a median $d^{\prime}$ of 1.55 (interquartile range 0.80 to 1.77) [Fig. 1(b)]. VSD fluorescence was imaged with 2-ms frame rates, and we found that the spatiotemporal $\Delta F / F_{0}$ responses were highly variable from trial to trial [Fig. 1(c)]. We quantified single-trial response amplitudes at the time of the peak of the average evoked response (within the first $100 \mathrm{~ms}$ after whisker deflection) relative to prestimulus fluorescence. The coefficient of variation in single-trial $\Delta F / F_{0}$ in $\mathrm{S} 1$ was median 0.71 (interquartile range 0.59 to 0.88 ), and in M1 was median 1.03 (interquartile range 0.77 to 1.35 ) across mice $(n=21)$. A similar high trial-to-trial variability of the amplitude of evoked postsynaptic potentials was previously reported in whole-cell membrane potential recordings from S1 during performance of a closely related whisker-detection task. ${ }^{33}$

\subsection{Hit Versus Miss Trials}

Our next goal was to investigate some of the behavioral variables correlating with trial-to-trial variability. We began this analysis by distinguishing hit trials in which the mouse licked after the whisker stimulus from miss trials in which the mouse did not lick after the whisker stimulus (Fig. 2). To help separate sensory and motor components, in this study, we specifically analyzed hit trials in which the reaction time was longer than $300 \mathrm{~ms}$. This included $71 \%$ of all hit trials, with $29 \%$ of hit trials being excluded because of reaction times below $300 \mathrm{~ms}$. However, analysis of all hit trials pooled together irrespective of reaction time gave essentially the same results.

In data from individual mice [Fig. 2(a)] and in the grand average data with images spatially aligned to the $\mathrm{C} 2$ whisker representation [Fig. 2(b)], the earliest components of the sensory response appeared to be similar between hit and miss trials. The initial response was a highly localized depolarization in $\mathrm{S} 1$ (see imaging frame at $16 \mathrm{~ms}$ after $\mathrm{C} 2$ whisker deflection). Over the next milliseconds, the response spread within $\mathrm{S} 1$ and initiated a secondary hotspot of depolarization in M1 (see imaging frame at $32 \mathrm{~ms}$ after $\mathrm{C} 2$ whisker deflection). The early response was quantified as the peak change in VSD fluorescence $\left(\Delta F / F_{0}\right)$ over the first $100 \mathrm{~ms}$ after $\mathrm{C} 2$ whisker deflection compared to the baseline fluorescence immediately before whisker stimulation averaged across trials. The ratio of the amplitude of the early sensory response for hit and miss trials was computed for each mouse, and median values were calculated across mice. The median hit-to-miss ratio for the early response in $\mathrm{S} 1$ was 1.19 (interquartile range 0.91 to 1.42 ), and for M1, it was 1.35 (interquartile range 0.93 to 1.72$)(n=21$ mice) [Fig. 2(c)]. On average, for each mouse, we analyzed 38 hit trials and 75 miss trials $(n=21$ mice).

A difference between hit and miss trials became obvious at times later than $100 \mathrm{~ms}$ after the whisker stimulus, with the VSD response distributed across a large area of the dorsal mouse neocortex, as seen in both individual mice [Fig. 2(a)] and the grand average [Fig. 2(b)] (see imaging frames at 150 and $250 \mathrm{~ms}$ after $\mathrm{C} 2$ whisker deflection). The late response was quantified as the change in fluorescence $\left(\Delta F / F_{0}\right)$ comparing the average over 100 to $300 \mathrm{~ms}$ with the prestimulus baseline averaged across trials. The median late hit-to-miss ratio was 1.81 (interquartile range 1.32 to 3.14 ) in $\mathrm{S} 1$ and 2.80 (interquartile range 2.06 to
3.73) in M1 [Fig. 2(c)]. The late hit-to-miss ratio was significantly larger than the early hit-to-miss ratio for both $\mathrm{S} 1$ $(P<0.0001)$ and M1 $(P<0.0001)$ [Fig. 2(c)].

We also computed the hit-to-miss ratio of the grand average VSD responses for each pixel at both early and late times [Fig. 2(d)]. The hit-to-miss ratio map indicated that the early evoked VSD response averaged over 10 to $50 \mathrm{~ms}$ after whisker stimulus was close to unity across the dorsal neocortex [Fig. 2(d)]. The hit-to-miss ratio computed across the grand average responses from 100 to $300 \mathrm{~ms}$ after whisker stimulus showed that late sensorimotor depolarization was considerably larger in hit trials than miss trials across the entire dorsal neocortex [Fig. 2(d)]. The late hit-to-miss ratio had a clear spatial pattern, with the smallest values in the neighborhood of S1 and the largest values in frontal regions. In particular, the highest hit-to-miss ratios appeared to localize to regions anterior, medial, and lateral to whisker M1. Thus, whereas the initial response of the dorsal neocortex showed little difference comparing hit and miss trials, at later times, the neocortex was much more depolarized in hit trials compared to miss trials, with the largest hit-related excitation in frontal cortical regions.

\subsection{Impact of Prestimulus Whisking on Behavioral Performance}

Ongoing whisker-related behavior has been shown to make a major impact upon responses evoked by whisker stimulation in previous membrane potential recordings of individual layer $2 / 3$ neurons $^{27,40}$ and also in previous VSD imaging studies. ${ }^{5,13}$ In a subset of experiments, we therefore filmed C2 whisker movements during task performance with a high-speed camera at $500 \mathrm{~Hz}$, matched frame by frame to the VSD imaging. The whisker movements were subsequently tracked offline by semiautomated procedures.

As shown in the example traces, prestimulus whisking was largely absent on hit trials but was prominent in some miss trials [Fig. 3(a)]. We quantified whisking as the standard deviation (SD) of whisker angle in $100 \mathrm{~ms}$ bins. The grand average SD of whisker angle was larger in miss than hit trials at all times quantified from immediately after the audiovisual cue (indicating trial initiation) until the time of the whisker stimulus $2.5 \mathrm{~s}$ later [Fig. 3(b)]. Hit trials therefore appeared to be associated with less whisker movement in the period between the cue and the whisker stimulus. For statistical analysis, we computed the SD of C2 whisker angle over the first $500 \mathrm{~ms}$ after cue presentation (postcue) and for the $500 \mathrm{~ms}$ immediately preceding the whisker stimulus (prestim) for each mouse, separating between hit and miss trials. Hit trials had a postcue median whisker angle SD of $1.35 \mathrm{deg}$ (interquartile range 0.99 to $2.04 \mathrm{deg}$ ). Miss trials had a postcue median whisker angle SD of 3.16 deg (interquartile range 2.13 to $3.37 \mathrm{deg}$ ). Hit trials had a prestim median whisker angle SD $0.74 \mathrm{deg}$ (interquartile range 0.66 to $0.81 \mathrm{deg}$ ). Miss trials had a prestim median whisker angle SD of $2.49 \mathrm{deg}$ (interquartile range 1.30 to $2.82 \mathrm{deg}$ ). Hit trials had significantly less whisking than miss trials in both postcue $(P=0.0002)$ and prestim $(P=0.0005)$ periods [Fig. 3(c)]. There was a significant reduction in whisking in the prestim period compared to the postcue period in both hit $(P=0.0002)$ and miss $(P=0.002)$ trials [Fig. 3(c)].

We classified trials into prestimulus whisking or prestimulus quiet (nonwhisking) using a threshold of $2 \mathrm{deg}$ of whisker angle 
(a) Experiment 140718 ( $n=37$ hit trials; $n=52$ miss trials)

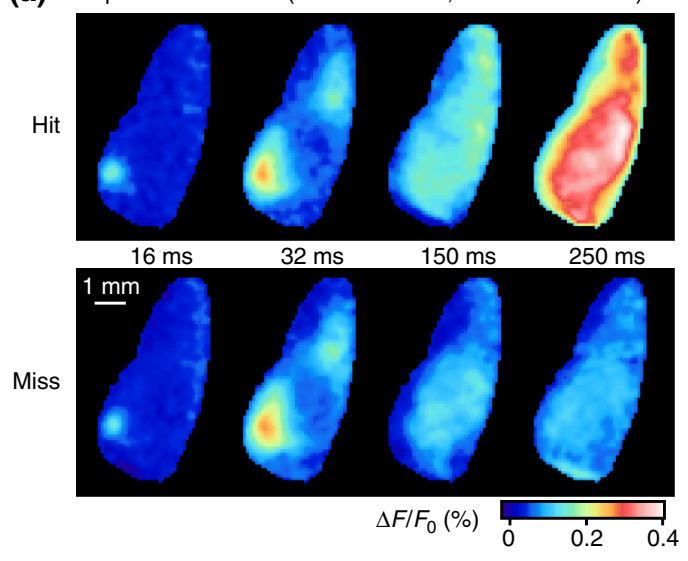

(b) Grand average $(n=21$ mice $)$
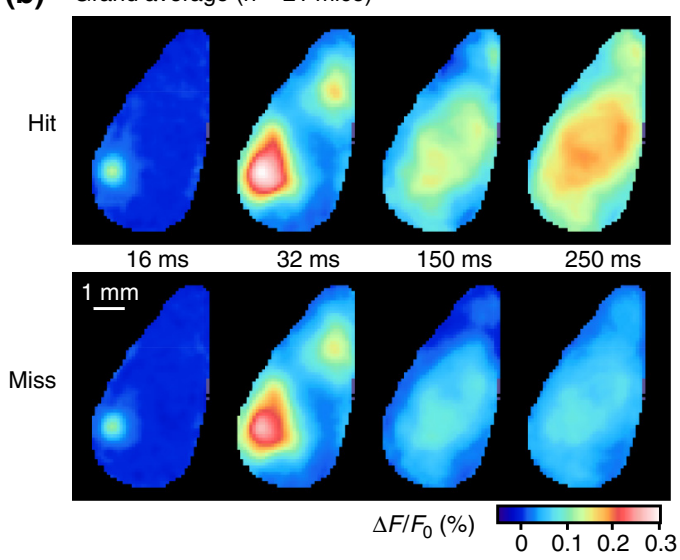

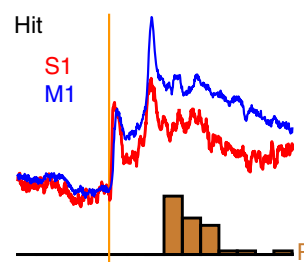

C2 whisker stimulus
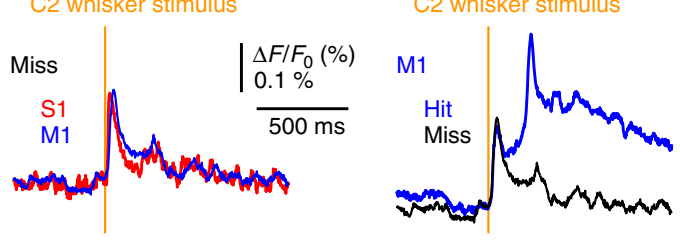

(c)

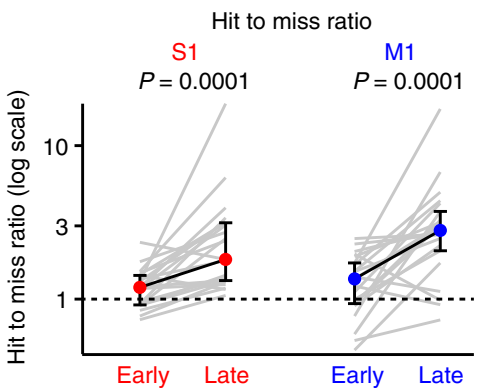

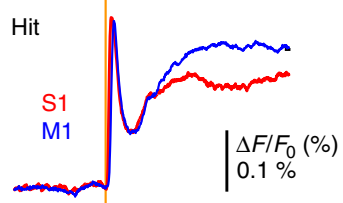

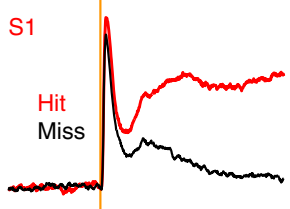

C2 whisker stimulus
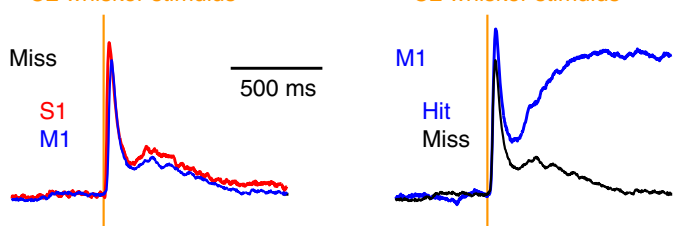

Fig. 2 VSD imaging of hit and miss trials during the detection task. (a) An example experiment showing the average of 37 hit trials (excluding trials with reaction times $<300 \mathrm{~ms}$ ) and an average of 52 miss trials. Images at different times postwhisker stimulus are shown for hit and miss trials (left), together with quantification of the time course of fluorescence changes (right) in regions of interest $\left(500 \times 500 \mu \mathrm{m}^{2}\right)$ centered on the $\mathrm{C} 2$ whisker responsive regions in S1 (red) and M1 (blue). Also shown as a histogram (brown) is the probability of licking at different poststimulus times. (b) Grand average of hit trials (excluding reaction times less than $300 \mathrm{~ms}$ ) and miss trials averaged across 21 mice. Because of differences in the size and shape of craniotomies, not all pixels are averages of the same number of mice (however, all pixels are averages of at least $n=18$ mice). (c) Ratio of evoked VSD responses for hit and miss trials computed for each individual experiment (gray lines, $n=21$ mice) separately for $\mathrm{S} 1$ and $\mathrm{M} 1$ for the early peak response within $100 \mathrm{~ms}$ of stimulus and a late period averaged from 100 to 300 ms after whisker stimulus. Median \pm interquartile range is shown in filled circles with error bars linked by a black line. The hit-to-miss ratio is significantly larger for the late component than for the early response in both $\mathrm{S} 1$ and M1 according to the Wilcoxon signed-rank test. (d) Ratio of the evoked VSD responses for the grand average of hit trials (excluding reaction times less than $300 \mathrm{~ms}$ ) divided by the grand average of miss trials. The "early" hit-to-miss ratio is computed for the average of image frames 10 to $50 \mathrm{~ms}$ after whisker stimulus. The "late" hit-to-miss ratio is computed for the average of image frames from 100 to $300 \mathrm{~ms}$ after whisker stimulus. Because of differences in the size and shape of craniotomies, not all pixels are averages of the same number of mice (however, all pixels are averages of at least $n=18$ mice). 


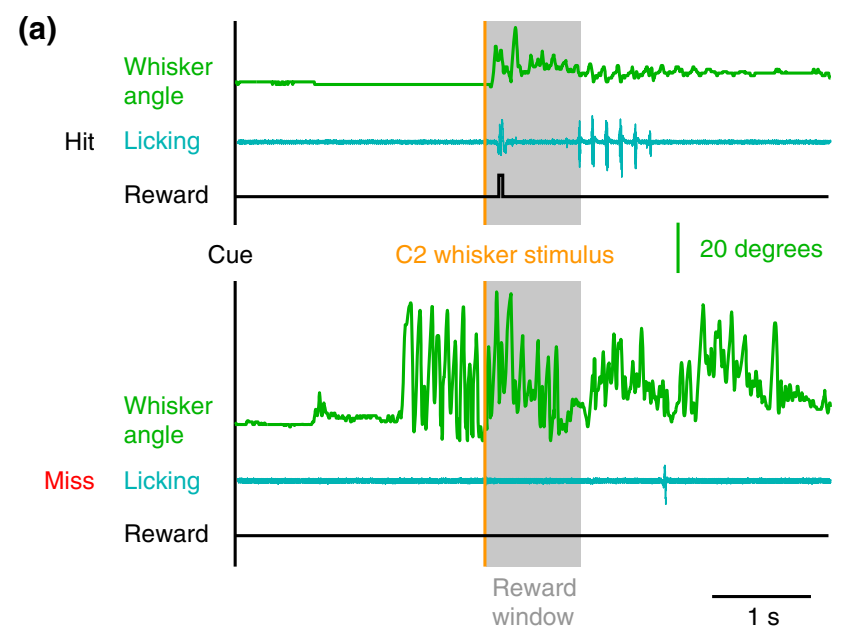

(c)

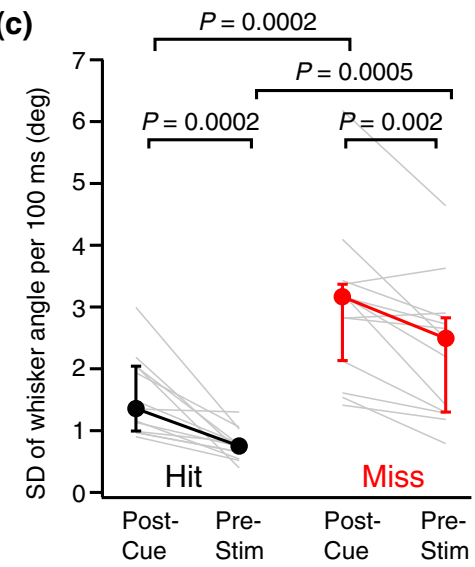

(b)
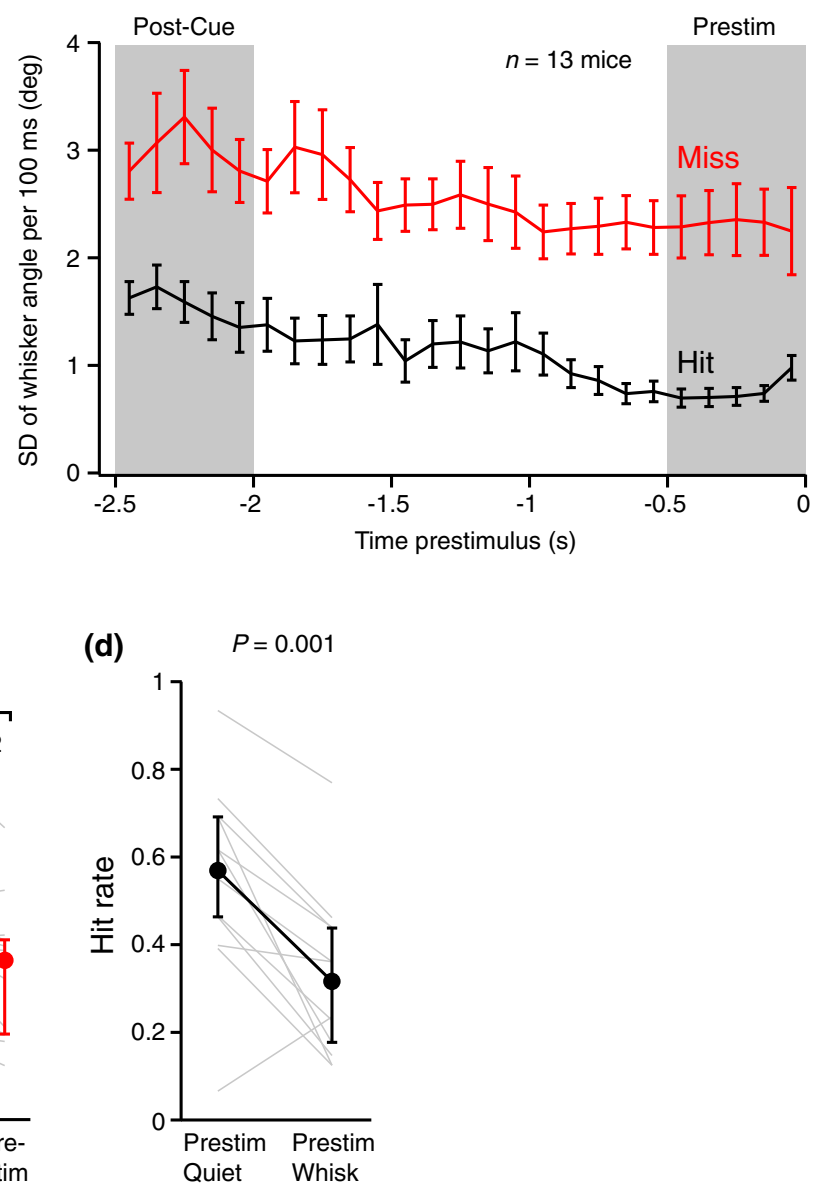

Fig. 3 Effect of prestimulus whisking on behavioral performance. (a) Example traces of whisker angle (obtained from analysis of high-speed video of whisker movements at $500 \mathrm{~Hz}$ ) and licking (obtained from piezo film attached to the water reward spout) together with timing of reward delivery for a hit trial (above) and a miss trial (below). Mice always moved their whiskers after stimulus in hit trials. In some trials, mice spontaneously moved their whiskers before stimulus. (b) The SD of the whisker angle was computed for $100-\mathrm{ms}$ bins from immediately after the cue at $-2.5 \mathrm{~s}$ until delivery of whisker stimulus at $0 \mathrm{~s}$ for hit and miss trials of each mouse. The SDs were then averaged across 13 mice and the mean values plotted together with SEM error bars. (c) The mean of five bins of the SD of the whisker angle from immediately after the cue (postcue: -2.5 to $-2.0 \mathrm{~s}$ ) and immediately before the whisker stimulus (prestim: -0.5 to $0 \mathrm{~s}$ ) for hit and miss trials. The data for each individual experiment are plotted in thin gray lines. Median \pm interquartile range is shown in filled circles with error bars linked by a thick line. For both hit and miss trials, there was a significant reduction in whisker movement between postcue and prestim periods (Wilcoxon signed-rank test). Miss trials had significantly more whisker movement than hit trials in both postcue and prestim periods (Wilcoxon signed-rank test). (d) Each trial was classified as prestim quiet or prestim whisking based on the SD of the whisker angle over the $500 \mathrm{~ms}$ immediately before whisker stimulus (the prestim period) with a threshold of $2 \mathrm{deg}$, then the hit rate was calculated for each case. The data for each mouse are shown in thin gray lines. Median \pm interquartile range is shown in filled circles with error bars linked by a thick line. The hit rate was significantly higher for prestim quiet compared to prestim whisking trials (Wilcoxon signedrank test).

SD in the 500-ms prestimulus period. Using this criterion, $29 \%$ of trials had prestimulus whisking. In prestimulus quiet trials, the median hit rate was $57 \%$ (interquartile range $46 \%$ to 69\%) [Fig. 3(d)]. In prestimulus whisking trials, the median hit rate was $32 \%$ (interquartile range $18 \%$ to $44 \%$ ) [Fig. 3(d)]. The hit rate in prestimulus whisking trials was significantly lower than the hit rate in prestimulus quiet trials $(P=0.001)$. Prestimulus whisking therefore appears to cause a substantial reduction in behavioral performance in this whisker-deflection detection task.

\subsection{Impact of Prestimulus Whisking on Voltage-Sensitive Dye Responses}

We next investigated the impact of prestimulus whisking on the evoked VSD responses, comparing hit and miss trials with and without prestimulus whisking (Fig. 4). On average, for each mouse, we analyzed 42 prestimulus quiet hit trials, 12 prestimulus whisking hit trials, 35 prestimulus quiet miss trials, and 19 prestimulus whisking miss trials $(n=13$ mice). The grand average early response over the first $100 \mathrm{~ms}$ after the $\mathrm{C} 2$ whisker 
(a)

Grand averages $(n=13$ mice $)$
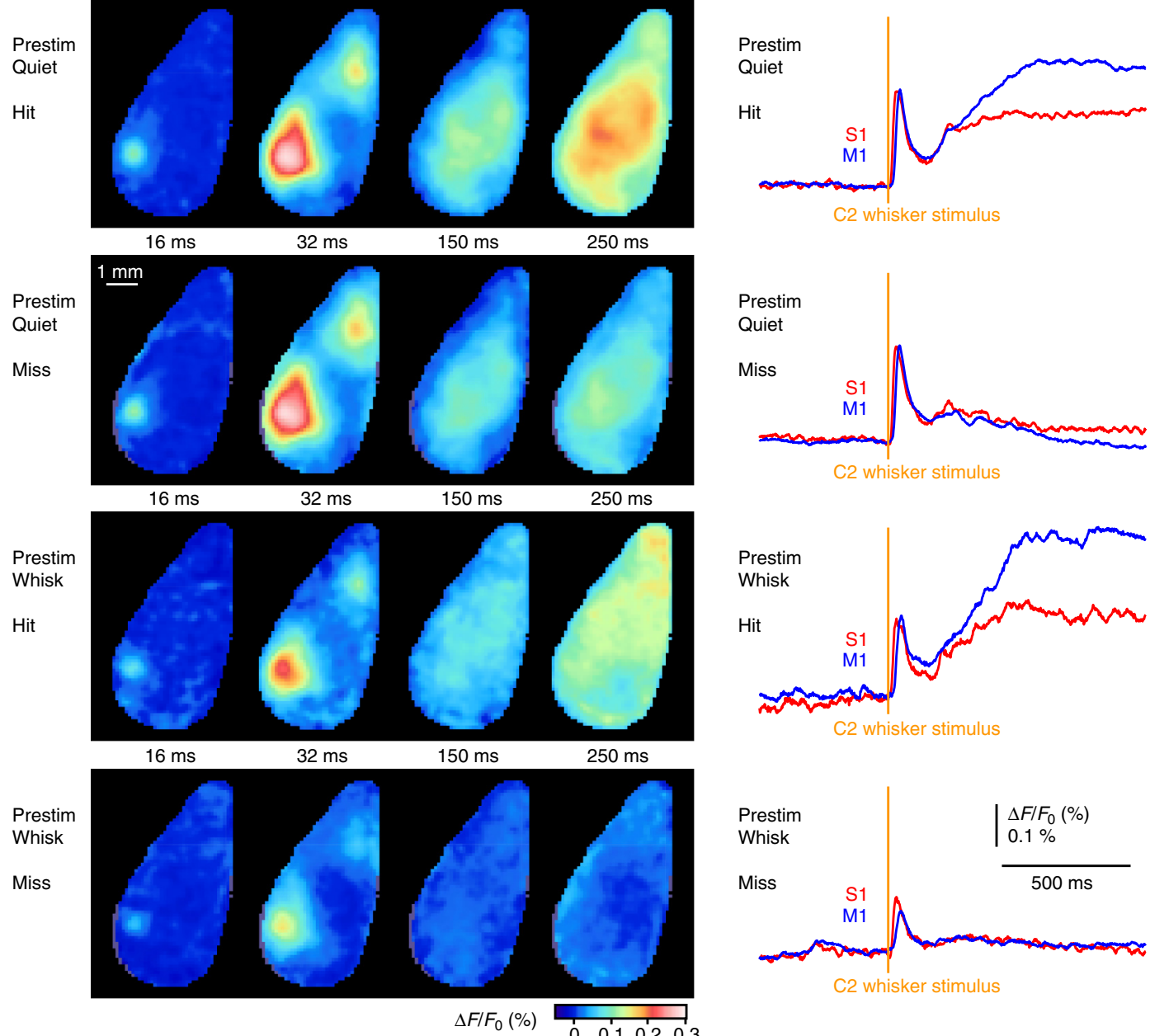

(b)

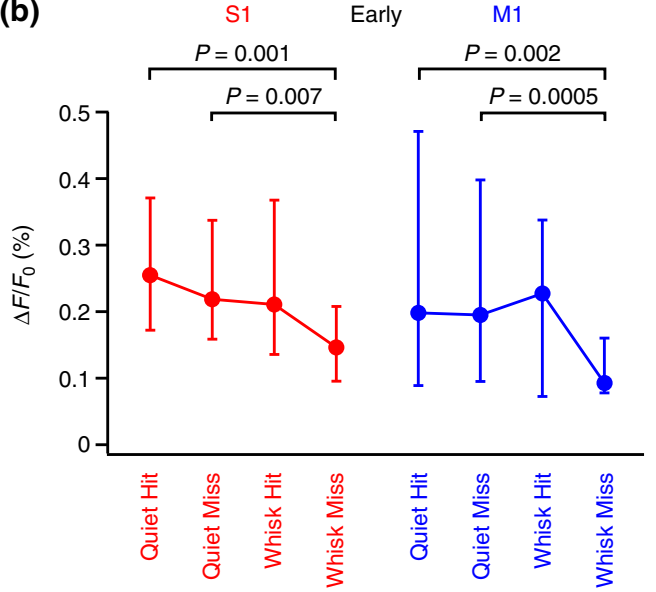

(c)

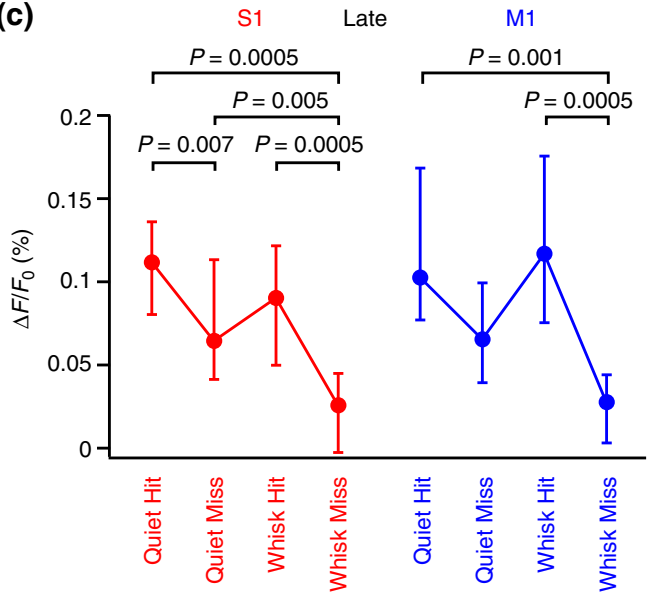

Fig. 4 Effect of prestimulus whisking on VSD responses. (a) Grand average images (left) and region of interest quantification for S1 and M1 (right) across 13 mice for different trial types depending upon prestimulus whisking and poststimulus licking: prestimulus quiet (no whisking) hit trials (top); prestimulus quiet miss trials (upper middle); prestimulus whisking hit trials (lower middle); and prestimulus whisking miss trials (bottom). (b) Quantification of the peak amplitude of the early sensory response in S1 and M1 showing median \pm interquartile range for the above trial conditions, together with statistically significant differences according to the Wilcoxon signed-rank test. (c) Quantification of the amplitude of late activity (100 to 300 ms poststimulus) in S1 and M1 showing median \pm interquartile range for the above trial conditions, together with statistically significant differences according to the Wilcoxon signed-rank test. 
Table 1 Prestimulus whisking versus quiet, and hit versus miss trials for S1 and M1 at early and late times. Data for Fig. 4.

\begin{tabular}{lcccc} 
Cortical region & Prestimulus quiet or whisking? & Hit or miss? & $\begin{array}{c}\text { Early peak } 0 \text { to } 100 \mathrm{ms:} \\
\text { median } \Delta F / F_{0}(\mathrm{IQR})\end{array}$ & $\begin{array}{c}\text { Average late } 100 \text { to } 300 \text { ms: } \\
\text { median } \Delta F / F_{0}(\text { IQR })\end{array}$ \\
\hline S1 & Quiet & Hit & $0.25 \%(0.17 \%$ to $0.37 \%)$ & $0.11 \%(0.08 \%$ to $0.14 \%)$ \\
S1 & Quiet & Miss & $0.22 \%(0.16 \%$ to $0.34 \%)$ & $0.06 \%(0.04 \%$ to $0.11 \%)$ \\
S1 & Whisking & Hit & $0.21 \%(0.14 \%$ to $0.37 \%)$ & $0.09 \%(0.05 \%$ to $0.12 \%)$ \\
S1 & Whisking & Miss & $0.15 \%(0.10 \%$ to $0.21 \%)$ & $0.03 \%(0.00 \%$ to $0.04 \%)$ \\
M1 & Quiet & Hit & $0.20 \%(0.09 \%$ to $0.47 \%)$ & $0.10 \%(0.08 \%$ to $0.17 \%)$ \\
M1 & Quiet & Miss & $0.19 \%(0.10 \%$ to $0.40 \%)$ & $0.07 \%(0.04 \%$ to $0.10 \%)$ \\
M1 & Whisking & Hit & $0.23 \%(0.07 \%$ to $0.34 \%)$ & $0.12 \%(0.08 \%$ to $0.18 \%)$ \\
M1 & Whisking & Miss & $0.09 \%(0.08 \%$ to $0.16 \%)$ & $0.03 \%(0.00 \%$ to $0.04 \%)$ \\
\hline
\end{tabular}

deflection appeared similar for prestimulus-quiet hit and prestimulus-quiet miss trials [Fig. 4(a)]. At later times, prestimulusquiet hit trials appeared to be more depolarized compared to prestimulus-quiet miss trials. Prestimulus-whisking trials in general appeared to have smaller-amplitude early responses, with prestimulus-whisking miss trials appearing to have responses of smaller amplitude and shorter duration with a lesser spatial extent. At later times, prestimulus-whisking hit trials appeared more depolarized than prestimulus-whisking miss trials.

We found that the early evoked response in S1 was significantly smaller in prestimulus-whisking miss trials compared to both prestimulus-quiet hit $(P=0.001)$ and prestimulus-quiet miss $(P=0.007)$ trials [Fig. 4(b)]. Similarly, the early evoked response in M1 was also significantly smaller in prestimuluswhisking miss trials compared to both prestimulus-quiet hit $(P=0.002)$ and prestimulus-quiet miss $(P=0.0005)$ trials [Fig. 4(b)]. The median values and interquartile ranges are given in Table 1.

In the late period of the sensorimotor response (100 to $300 \mathrm{~ms}$ after whisker stimulus), there were many significant differences in S1 comparing the four trial types [Fig. 4(c)]. Hit trials had significantly larger late depolarization compared to miss trials for both prestimulus-quiet $(P=0.007)$ and prestimulus-whisking $(P=0.0005)$ conditions. Miss trials with prestimulus-whisking had significantly less late depolarization than both hit $(P=0.0005)$ and miss $(P=0.005)$ prestimulusquiet trials. The late response in M1 was significantly smaller in prestimulus-whisking miss trials compared to hit trials of both prestimulus-whisking $(P=0.0005)$ and prestimulus-quiet $(P=0.001)$ trial types. The median values and interquartile ranges are given in Table 1.

Prestimulus whisking correlates with increased miss rates, and, in these miss trials, small responses are evoked by $\mathrm{C} 2$ whisker deflection at both early and late times in both S1 and M1.

\subsection{Poststimulus Whisking}

In a previous study of a closely related whisker detection task, mice were found to protract their whiskers rhythmically after the whisker stimulus in all hit trials. ${ }^{33}$ However, in prestimulus-quiet miss trials, the mouse does not necessarily make whisker movements after the whisker stimulus. We therefore compared three trial types of VSD responses: (i) prestimulus-quiet hit trials in which the mouse would always initiate poststimulus whisking, (ii) prestimulus-quiet miss trials in which the mouse would initiate poststimulus whisking, and (iii) prestimulus-quiet miss trials in which the mouse remained quiet (not moving its whiskers) after the whisker stimulus. On average, for each mouse, we analyzed prestimulus quiet trials for 42 hit trials with poststimulus whisking, 20 miss trials with poststimulus whisking, and 15 miss trials without poststimulus whisking ( $n=13$ mice). The grand average responses appeared to indicate that poststimulusquiet miss trials were accompanied by smaller, shorter, and more localized sensory responses compared to poststimulus-whisking trials [Fig. 5(a)]. The largest early responses appeared to occur in miss trials with poststimulus whisking, whereas the largest late responses appeared to occur in hit trials [Fig. 5(a)].

For the early sensory response in S1, statistical analysis revealed that poststimulus-quiet miss trials had significantly smaller responses than hit trials $(P=0.01)$. In M1, the early sensory response was significantly larger in trials with poststimulus whisking compared to poststimulus-quiet trials (hit with poststimulus whisking versus miss with poststimulus quiet, $P=0.004$; miss with poststimulus whisking versus miss with poststimulus quiet, $P=0.008$ ) [Fig. 5(b)]. The median values and interquartile ranges are given in Table 2.

At late times (100 to $300 \mathrm{~ms}$ after whisker stimulus), prestimulus-quiet miss trials without poststimulus whisking had significantly smaller depolarization compared to hit trials in both $\mathrm{S} 1(P=0.004)$ and M1 $(P=0.004)$ [Fig. 5(c)]. The median values and interquartile ranges are given in Table 2 .

The presence or absence of poststimulus whisking in miss trials therefore correlates with important differences in the spatiotemporal dynamics of cortical activity.

\section{Discussion}

Through VSD imaging of the dorsal mouse neocortex, we found highly distributed and dynamic sensorimotor processing of a whisker stimulus and its conversion into licking motor output for reward. Sensorimotor processing differed strongly during late periods of hit versus miss trials. Prestimulus whisking suppressed sensorimotor responses to whisker stimulation and reduced hit rates. In some trials, the mouse did not lick or move its whiskers, and these trials also had small responses to whisker stimulation. Our analysis therefore begins to reveal behavioral correlates of sensorimotor processing in dorsal mouse neocortex during execution of a simple whisker detection task. 
(a) Grand averages $(n=13$ mice $)$
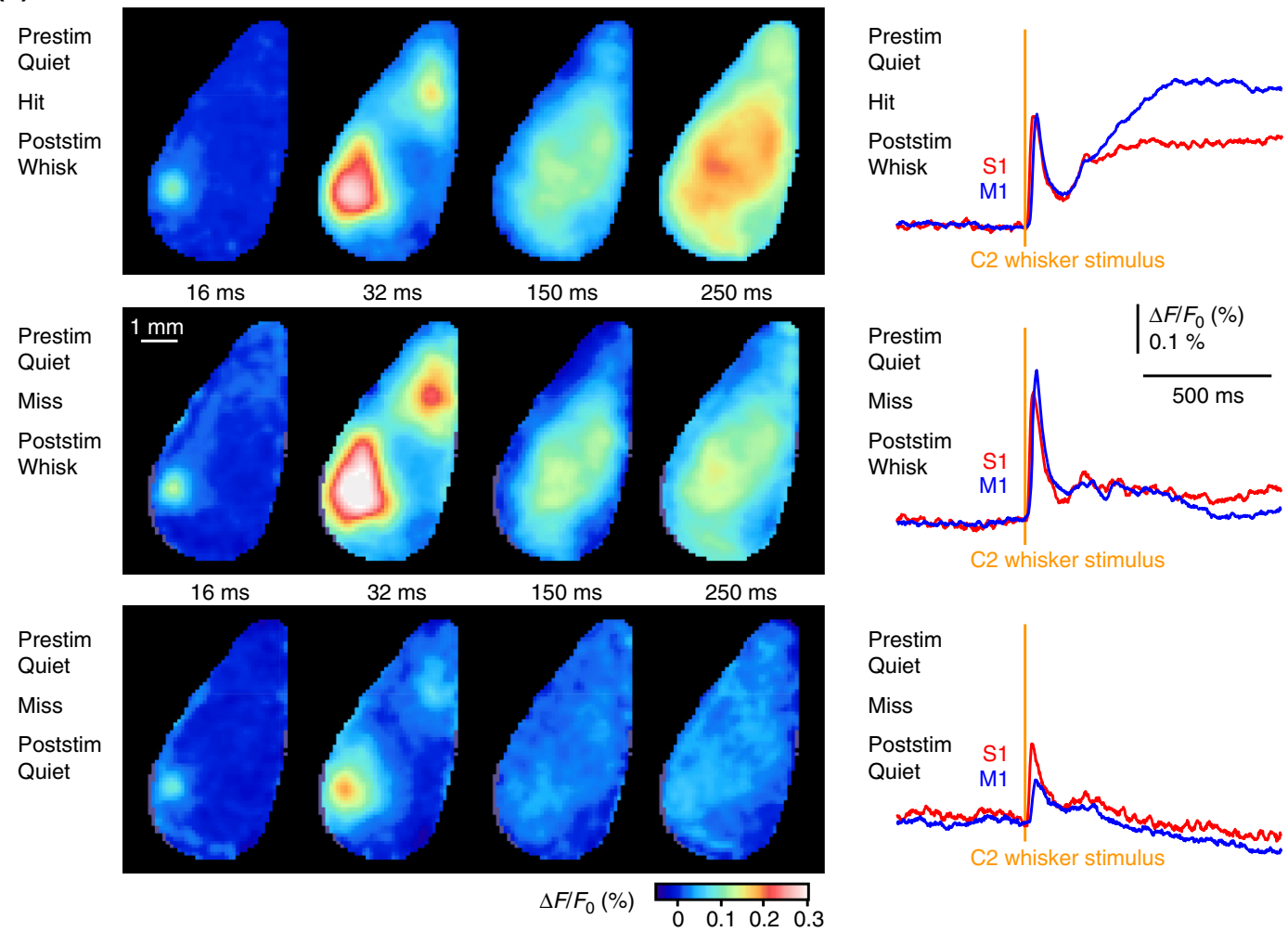

(b)

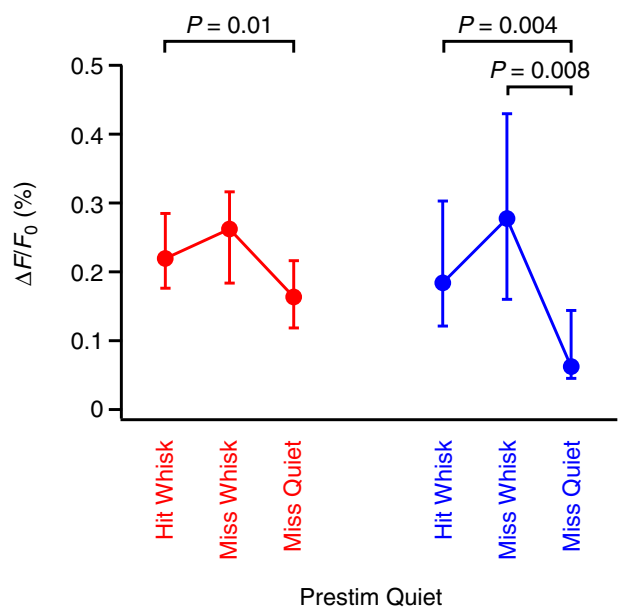

(c)

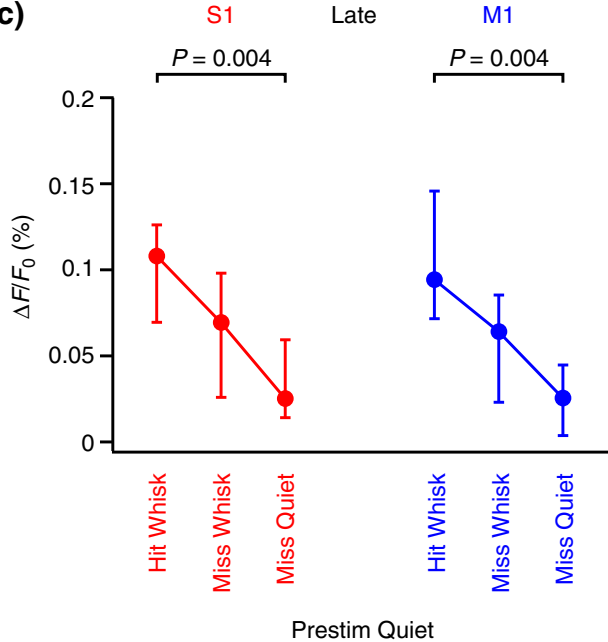

Fig. 5 Effect of poststimulus whisking on VSD responses. (a) Grand average images (left) and region of interest quantification for $\mathrm{S} 1$ and $\mathrm{M} 1$ (right) across 13 mice for different trial types depending upon the presence or absence of poststimulus whisking and licking. Here, we selected for prestimulus quiet (no whisking) trials with: hit trials, which were always accompanied by poststimulus whisking (top); miss trials with poststimulus whisking (middle); and miss trials without poststimulus whisking (poststimulus quiet) (bottom). (b) Quantification of the peak amplitude of the early sensory response in S1 and M1 showing median \pm interquartile range for the above trial conditions, together with statistically significant differences according to the Wilcoxon signed-rank test. (c) Quantification of the amplitude of late activity (100 to 300 ms poststimulus) in S1 and M1 showing median \pm interquartile range for the above trial conditions, together with statistically significant differences according to the Wilcoxon signed-rank test.

\subsection{Comparison with Naïve Mice}

The spatiotemporal dynamics of the early VSD response over the first $100 \mathrm{~ms}$ after deflection of the $\mathrm{C} 2$ whisker during task performance in mice after learning was qualitatively similar to previous results obtained in naïve awake mice adapted to head restraint but not carrying out any task. ${ }^{13}$ In naïve mice, we found early localized responses in S1, which spread within S1 and S2, followed by excitation of the frontal wM1 region, ${ }^{13}$ similar to the pattern of activity observed here in task-performing mice (Fig. 2). Furthermore, sensory responses were reduced in amplitude when naïve mice were whisking during stimulus delivery, ${ }^{13}$ 
Table 2 Poststimulus whisking versus quiet, and hit versus miss trials for S1 and M1 at early and late times. Data for Fig. 5.

\begin{tabular}{lccccc} 
Cortical region & $\begin{array}{c}\text { Prestimulus quiet } \\
\text { or whisking? }\end{array}$ & Hit or miss? & $\begin{array}{c}\text { Poststimulus quiet } \\
\text { or whisking? }\end{array}$ & $\begin{array}{c}\text { Early peak } 0 \text { to } 100 \text { ms: } \\
\text { median } \Delta F / F_{0} \text { (IQR) }\end{array}$ & $\begin{array}{c}\text { Average late } 100 \text { to } 300 \text { ms: } \\
\text { median } \Delta F / F_{0}(\text { IQR) }\end{array}$ \\
\hline S1 & Quiet & Hit & Whisking & $0.22 \%(0.18 \%$ to $0.28 \%)$ & $0.11 \%(0.07 \%$ to $0.13 \%)$ \\
S1 & Quiet & Miss & Whisking & $0.26 \%(0.18 \%$ to $0.32 \%)$ & $0.07 \%(0.03 \%$ to $0.10 \%)$ \\
S1 & Quiet & Miss & Quiet & $0.16 \%(0.12 \%$ to $0.22 \%)$ & $0.03 \%(0.01 \%$ to $0.06 \%)$ \\
M1 & Quiet & Hit & Whisking & $0.18 \%(0.12 \%$ to $0.30 \%)$ & $0.09 \%(0.07 \%$ to $0.15 \%)$ \\
M1 & Quiet & Miss & Whisking & $0.28 \%(0.16 \%$ to $0.43 \%)$ & $0.06 \%(0.02 \%$ to $0.09 \%)$ \\
M1 & Quiet & Miss & Quiet & $0.06 \%(0.05 \%$ to $0.14 \%)$ & $0.03 \%(0.00 \%$ to $0.04 \%)$ \\
\hline
\end{tabular}

just as we observed here in the task-performing mice (Fig. 4). When naïve mice actively moved their whiskers immediately after whisker deflection, there were larger responses in both S1 and M1 compared to trials without such sensory-evoked whisker movements. ${ }^{13}$ Similarly, in task-performing mice, there was also more activity across the neocortex in trials with sensory-evoked licking (Fig. 2). However, at times longer than $100 \mathrm{~ms}$ after whisker deflection, the VSD responses of naïve and trained mice were qualitatively different, with only trained mice exhibiting the prolonged highly distributed licking-related activity in hit trials across sensorimotor cortex.

\subsection{Differential Sensorimotor Processing in Hit Versus Miss Trials}

During the VSD imaging, the 1-ms deflection of the C2 whisker evoked licking to obtain a reward in $41 \%$ of trials, whereas in trials without whisker stimulus, mice only licked on $7 \%$ of trials for which they were not rewarded. Mice therefore discriminated trials with and without whisker stimulus, giving rise to a $d^{\prime}$ of 1.55. The largest differences between the VSD responses in hit and miss trials were at late times, more than $100 \mathrm{~ms}$ after whisker stimulus [Fig. 2(c)]. This is in agreement with previous data from membrane potential recordings in S1 during closely related whisker detection tasks. ${ }^{33,34,36}$ Within the imaged portion of the contralateral dorsal neocortex, the largest hit versus miss differences were located in the frontal cortex in regions surrounding whisker M1, located at $\sim 1 \mathrm{~mm}$ anterior and $\sim 1 \mathrm{~mm}$ lateral to Bregma [Fig. 2(d)]. The frontal region showing strong hit versus miss differences may include a neocortical region involved in goal-directed licking, the anterior lateral motor cortex, which is located at $\sim 2.5 \mathrm{~mm}$ anterior and $\sim 1.5 \mathrm{~mm}$ lateral to Bregma, ${ }^{41,42}$ although this region was often located close to the edge of our craniotomy. The quantification of the late response was carried out from 100 to $300 \mathrm{~ms}$, preceding the 300-ms minimum first lick response time included in our analyses. The excitation of frontal tongue/jaw-related motor/ premotor areas might therefore causally contribute to driving the licking motor output in our task, and future experiments should directly investigate these cortical regions in detail. However, it is important to note that all regions of the dorsal neocortex included in our imaging experiments were more depolarized in general in hit compared to miss trials, so many cortical regions might contribute to generating the licking motor command necessary for hit trials. A key future experiment will therefore be to inactivate many different cortical regions and examine the impact upon task performance, as recently carried out in a whisker object localization task..$^{41,43}$ It will be equally important to investigate where in the cortex one can stimulate to substitute for whisker stimulation in our whisker detection task. So far, it has been reported that optogenetic stimulation of S1 can substitute for whisker stimulation in both learning and execution of the task. ${ }^{33}$ It will be of great interest to quantitatively map the ability to evoke licking across many neocortical areas in trained mice. Such future experiments will suggest necessary and sufficient maps of cortical activity for performing the detection task, which would form an important complement to the correlational data of this study.

\subsection{Whisking-Related Modulation of Behavioral Performance and Sensorimotor Processing}

Previous studies have noted that passive whisker deflections give rise to bigger sensory evoked responses in S1 when the animal is in a quiet state without whisker movements compared to the response evoked by the same peripheral stimulus delivered when the animal is actively moving its whiskers. ${ }^{5,13,27,40,44}$ Our results now further reveal that whisking appears to reduce performance in a whisker stimulus detection task with perceived stimuli reported through goal-directed licking. The hit rate for prestimulus quiet trials was $57 \%$, but in prestimulus whisking trials, it was reduced to only 32\% [Fig. 3(d)]. Mice reduced whisking in the $500-\mathrm{ms}$ prestimulus period compared to the 500-ms postcue period [Fig. 3(c)], perhaps enhancing stimulus perception, and suggesting that the audiovisual cue could have provided the mouse with information about the timing of expected whisker stimuli. It is also possible that the audiovisual cue itself induced transient whisking, but we cannot directly address this since we did not film whisker movements before cue onset. In the future, it will be of great interest to explicitly manipulate cue timing and saliency and study the effects upon psychophysical thresholds for performance in the detection task across different whisker stimulus intensities.

Prestimulus whisking increased the fraction of miss trials. In these prestimulus whisking miss trials, the early sensory evoked response in both S1 and M1 was small in amplitude, duration, and spatial extent (Fig. 4). The increased probability of failing to lick in response to whisker stimulus in prestimulus whisking trials may therefore result from the weaker depolarization being unable to drive sufficient numbers of action potentials in the appropriate neurons in the neuronal networks underlying the sensorimotor transformation of whisker stimulus into licking motor output. 
That prestimulus whisking trials had smaller amplitude responses and were associated with decreased hit rates likely contributes to the grand average hit versus miss comparison (Fig. 2), in which the early response over the first $100 \mathrm{~ms}$ after the $\mathrm{C} 2$ whisker stimulus was slightly larger on average in hit compared to miss trials, with a $19 \%$ larger early response in S1 and a 35\% larger early response in M1. Similarly, a slightly larger early sensory-evoked response in hit trials compared to miss trials was previously reported in single-cell electrophysiological measurements from S1 during similar whisker detection tasks. ${ }^{34,36}$ Prestimulus whisking might also have contributed to miss trials in these previous studies. However, there are likely to be many different ways in which the neuronal networks contributing to the transformation of the whisker stimulus into a licking motor command might fail in miss trials.

\subsection{Voltage-Sensitive Dye Imaging-Technical Considerations}

In this study, we applied VSD RH1691 to the dorsal mouse neocortex to image cortical spatiotemporal dynamics during task performance. This technique provides interesting data on the spatiotemporal dynamics of cortical activity on the millisecond timescale. However, VSD imaging as applied in this study also has many limitations. Most importantly, the technique is highly invasive, requiring large craniotomies and long staining periods after dura removal. The difficulty in obtaining a good preparation for imaging and also subsequently having good performance in the detection task severely limited our dataset. Mice appeared to be less motivated to lick during the imaging session compared to the previous training day, with reduced hit rates and reduced false-alarm rates, which resulted in little change to $d^{\prime}$. In the future, it will be of great interest to image cortical dynamics with genetically encoded fluorescent voltage sensors, which have recently been improving significantly. ${ }^{45-48}$ Such genetically encoded sensors would allow longitudinal imaging during task learning and measurement from specific genetically defined populations of neurons. In the near future, it is therefore likely that we will learn much more about the spatiotemporal dynamics of cortical activity in specific cell types during specific behaviors.

\section{Conclusion}

Large-areas of dorsal neocortex depolarize in a highly dynamic spatiotemporal pattern during the execution of a simple goaldirected sensorimotor task in which a mouse converts the 1-ms deflection of the $\mathrm{C} 2$ whisker into licking motor output to receive a water reward. Future experiments must investigate in which way these patterns of neocortical activity participate causally in task execution and learning.

\section{Acknowledgments}

This work was funded by grants from the European Molecular Biology Organization (AK), Human Frontier Science Program Long-Term Fellowship (AK), Human Frontier Science Program Grant (CCHP), Swiss National Science Foundation (CCHP), SystemsX.ch (CCHP), and European Research Council (CCHP). The authors declare that they have no conflicts of interest.

\section{References}

1. D. Shoham et al., "Imaging cortical dynamics at high spatial and temporal resolution with novel blue voltage-sensitive dyes," Neuron $\mathbf{2 4}$, 791-802 (1999)

2. A. Grinvald and R. Hildesheim, "VSDI: a new era in functional imaging of cortical dynamics," Nat. Rev. Neurosci. 5, 874-885 (2004).

3. C. C. H. Petersen, A. Grinvald, and B. Sakmann, "Spatiotemporal dynamics of sensory responses in layer $2 / 3$ of rat barrel cortex measured in vivo by voltage-sensitive dye imaging combined with whole-cell voltage recordings and anatomical reconstructions," J. Neurosci. 23, 1298-1309 (2003).

4. C. C. H. Petersen et al., "Interaction of sensory responses with spontaneous depolarization in layer $2 / 3$ barrel cortex," Proc. Natl. Acad. Sci. U. S. A. 100, 13638-13643 (2003).

5. I. Ferezou, S. Bolea, and C. C. H. Petersen, "Visualizing the cortical representation of whisker touch: voltage-sensitive dye imaging in freely moving mice," Neuron 50, 617-629 (2006).

6. T. Berger et al., "Combined voltage and calcium epifluorescence imaging in vitro and in vivo reveals subthreshold and suprathreshold dynamics of mouse barrel cortex," J. Neurophysiol. 97, 3751-3762 (2007).

7. C. C. H. Petersen, "The functional organisation of the barrel cortex," Neuron 56, 339-355 (2007).

8. D. Feldmeyer et al., "Barrel cortex function," Prog. Neurobiol. 103, 3-27 (2013).

9. H. S. Orbach, L. B. Cohen, and A. Grinvald, "Optical mapping of electrical activity in rat somatosensory and visual cortex," J. Neurosci. 5, 1886-1895 (1985)

10. D. Kleinfeld and K. R. Delaney, "Distributed representation of vibrissa movement in the upper layers of somatosensory cortex revealed with voltage-sensitive dyes," J. Comp. Neurol. 375, 89-108 (1996).

11. D. Derdikman et al., "Imaging spatiotemporal dynamics of surround inhibition in the barrels somatosensory cortex," J. Neurosci. 23, 3100-3105 (2003).

12. E. F. Civillico and D. Contreras, "Integration of evoked responses in supragranular cortex studied with optical recordings in vivo," J. Neurophysiol. 96, 336-351 (2006).

13. I. Ferezou et al., "Spatiotemporal dynamics of cortical sensorimotor integration in behaving mice," Neuron 56, 907-923 (2007).

14. M. H. Mohajerani et al., "Mirrored bilateral slow-wave cortical activity within local circuits revealed by fast bihemispheric voltage-sensitive dye imaging in anesthetized and awake mice," J. Neurosci. 30, 3745-3751 (2010)

15. F. Matyas et al., "Motor control by sensory cortex," Science 330, 1240 1243 (2010).

16. M. H. Mohajerani et al., "Spontaneous cortical activity alternates between motifs defined by regional axonal projections," Nat. Neurosci. 16, 1426-1435 (2013).

17. D. R. Ollerenshaw et al., "The adaptive trade-off between detection and discrimination in cortical representations and behavior," Neuron 81, 1152-1164 (2014).

18. D. C. Millard et al., "Electrical and optical activation of mesoscale neural circuits with implications for coding," J. Neurosci. 35, 15702-15715 (2015).

19. H. J. Zheng, Q. Wang, and G. B. Stanley, "Adaptive shaping of cortical response selectivity in the vibrissa pathway," J. Neurophysiol. 113 3850-3865 (2015)

20. S. Manita et al., "A top-down cortical circuit for accurate sensory perception,” Neuron 86, 1304-1316 (2015)

21. A. J. Borgdorff, J. F. A. Poulet, and C. C. H. Petersen, "Facilitating sensory responses in developing mouse somatosensory barrel cortex," J. Neurophysiol. 97, 2992-3003 (2007).

22. J. W. Yang et al., "Thalamic network oscillations synchronize ontogenetic columns in the newborn rat barrel cortex," Cereb. Cortex 23, 1299-1316 (2013)

23. E. L. White and R. A. DeAmicis, "Afferent and efferent projections of the region of mouse SmI cortex which contains the posteromedial barrel subfield," J. Comp. Neurol. 175, 455-481 (1977).

24. E. Welker, P. V. Hoogland, and H. Van der Loos, "Organization of feedback and feedforward projections of the barrel cortex: a PHA-L study in the mouse," Exp. Brain Res. 73, 411-435 (1988).

25. R. Aronoff et al., "Long-range connectivity of mouse primary somatosensory barrel cortex," Eur. J. Neurosci. 31, 2221-2233 (2010). 
26. T. Mao et al., "Long-range neuronal circuits underlying the interaction between sensory and motor cortex," Neuron 72, 111-123 (2011).

27. T. Yamashita et al., "Membrane potential dynamics of neocortical projection neurons driving target-specific signals," Neuron 80, 1477-1490 (2013).

28. C. C. H. Petersen, "Cortical control of whisker movement," Аnпи. Rev. Neurosci. 37, 183-203 (2014).

29. V. Sreenivasan et al., "Parallel pathways from motor and somatosensory cortex for controlling whisker movements in mice," Eur. J. Neurosci. 41, 354-367 (2015).

30. J. C. Erlich, M. Bialek, and C. D. Brody, "A cortical substrate for memory-guided orienting in the rat," Neuron 72, 330-343 (2011).

31. D. Huber et al., "Multiple dynamic representations in the motor cortex during sensorimotor learning," Nature 484, 473-478 (2012).

32. V. Y. Cao et al., "Motor learning consolidates Arc-expressing neuronal ensembles in secondary motor cortex," Neuron 86, 1385-1392 (2015).

33. S. Sachidhanandam et al., "Membrane potential correlates of sensory perception in mouse barrel cortex," Nat. Neurosci. 16, 1671-1677 (2013).

34. H. Yang et al., "Origins of choice-related activity in mouse somatosensory cortex," Nat. Neurosci. 19, 127-134 (2016).

35. S. Sachidhanandam, B. S. Sermet, and C. C. H. Petersen, "Parvalbuminexpressing GABAergic neurons in mouse barrel cortex contribute to gating a goal-directed sensorimotor transformation," Cell Reports 15, 700-706 (2016).

36. T. Yamashita and C. C. H. Petersen, "Target-specific membrane potential dynamics of neocortical projection neurons during goal-directed behavior," eLife 5, e15798 (2016).

37. E. Zagha, X. Ge, and D. A. McCormick, "Competing neural ensembles in motor cortex gate goal-directed motor output," Neuron 88, 565-577 (2015).

38. T. Sippy et al., "Cell-type-specific sensorimotor processing in striatal projection neurons during goal-directed behavior," Neuron 88, 298305 (2015).

39. T. Miyashita and D. Feldman, "Behavioral detection of passive whisker stimuli requires somatosensory cortex," Cereb. Cortex 23, 1655-1662 (2013).

40. S. Crochet and C. C. H. Petersen, "Correlating whisker behavior with membrane potential in barrel cortex of awake mice," Nat. Neurosci. 9, 608-610 (2006).

41. Z. V. Guo et al., "Flow of cortical activity underlying a tactile decision in mice," Neuron 81, 179-194 (2014).

42. N. Li et al., "A motor cortex circuit for motor planning and movement," Nature 519, 51-56 (2015).

43. S. Crochet and C. C. H. Petersen, "From perception to action: a spatiotemporal cortical map," Neuron 81, 5-8 (2014).

44. H. Hentschke, F. Haiss, and C. Schwarz, "Central signals rapidly switch tactile processing in rat barrel cortex during whisker movements," Cereb. Cortex 16, 1142-1156 (2006).

45. W. Akemann et al., "Imaging brain electric signals with genetically targeted voltage-sensitive fluorescent proteins," Nat. Methods 7, 643-649 (2010).
46. L. Jin et al., "Single action potentials and subthreshold electrical events imaged in neurons with a fluorescent protein voltage probe," Neuron 75, 779-785 (2012).

47. D. R. Hochbaum et al., "All-optical electrophysiology in mammalian neurons using engineered microbial rhodopsins," Nat. Methods 11, 825-833 (2014)

48. Y. Gong et al., "High-speed recording of neural spikes in awake mice and flies with a fluorescent voltage sensor," Science 350, 1361-1366 (2015).

Alexandros Kyriakatos obtained his Bachelor of Arts and Sciences in psychology from the American College of Greece. He obtained his $\mathrm{PhD}$ in neuroscience from Karolinska Institute, Sweden, investigating neuromodulation of spinal locomotor networks. As a postdoctoral fellow, he then joined the laboratory of Prof. Carl Petersen at the Ecole Polytechnique Federale de Lausanne (EPFL) in Switzerland to investigate cortical sensorimotor processing in mice using voltage-sensitive dye imaging.

Vijay Sadashivaiah received his bachelor's degree in electrical engineering from PES University (India) in 2015. He participated in the Summer Research Program at the Ecole Polytechnique Federale de Lausanne in Switzerland and afterward joined the Graduate School of Biomedical Engineering at Johns Hopkins University, where he is currently a master's student. He is an active member of the Biomedical Engineering Society and Institute of Electrical and Electronics Engineers.

Yifei Zhang received his Bachelor of Engineering degree from Tsinghua University, China, in 2013, and his Master of Science degree from the EPFL, Switzerland, in 2016.

Alessandro Motta received his bachelor's degree in 2014 and his master's degree in 2016, both in life sciences from EPFL, Switzerland.

Matthieu Auffret is a PhD student in the Laboratory of Sensory Processing headed by Prof. Carl Petersen at the EPFL, Switzerland. His work focuses on large-scale sensory-motor integration in mouse cortex. He received his bachelor's degree in life sciences in 2011 and his master's degree in bioengineering in 2013 , both from the EPFL, Switzerland. He performed his master's project at Harvard University studying low-field MRI.

Carl C. H. Petersen is professor of neuroscience at the EPFL, Switzerland, where he investigates sensory perception in mice using electrophysiological and optical techniques. He investigated calcium signaling during his $\mathrm{PhD}$ in Cambridge, UK, supervised by Prof. Sir Michael Berridge. Then he trained in neurophysiology with Prof. Roger Nicoll at the University of California San Francisco, USA, and Prof. Bert Sakmann at the Max Planck Institute for Medical Research in Heidelberg, Germany. 